\title{
Correction to: Deep learning reconstruction improves image quality of abdominal ultra-high-resolution $\mathrm{CT}$
}

\author{
Motonori Akagi ${ }^{1} \cdot$ Yuko Nakamura $^{1} \cdot$ Toru Higaki $^{1} \cdot$ Keigo Narita $^{1} \cdot$ Yukiko Honda $^{1} \cdot$ Jian Zhou ${ }^{2} \cdot$ Zhou Yu $^{2}$. \\ Naruomi Akino $^{3} \cdot$ Kazuo Awai $^{1}$
}

Published online: 27 May 2019

(C) European Society of Radiology 2019

\section{Correction to: European Radiology}

https://doi.org/10.1007/s00330-019-06170-3

The original version of this article, published on 11 April 2019, unfortunately, contained a mistake. The following correction has therefore been made in the original: The image in Fig. $3 \mathrm{c}$ was wrong. The corrected figure is given below. The original article has been corrected.

The online version of the original article can be found at https://doi.org/ 10.1007/s00330-019-06170-3

Yuko Nakamura

yukon@hiroshima-u.ac.jp

1 Diagnostic Radiology, Hiroshima University, 1-2-3 Kasumi,

Minami-ku, Hiroshima, Japan

2 Canon Medical Research USA, Inc., Vernon Hills, IL, USA

3 Canon Medical Systems Co. Ltd., Otawara, Japan 

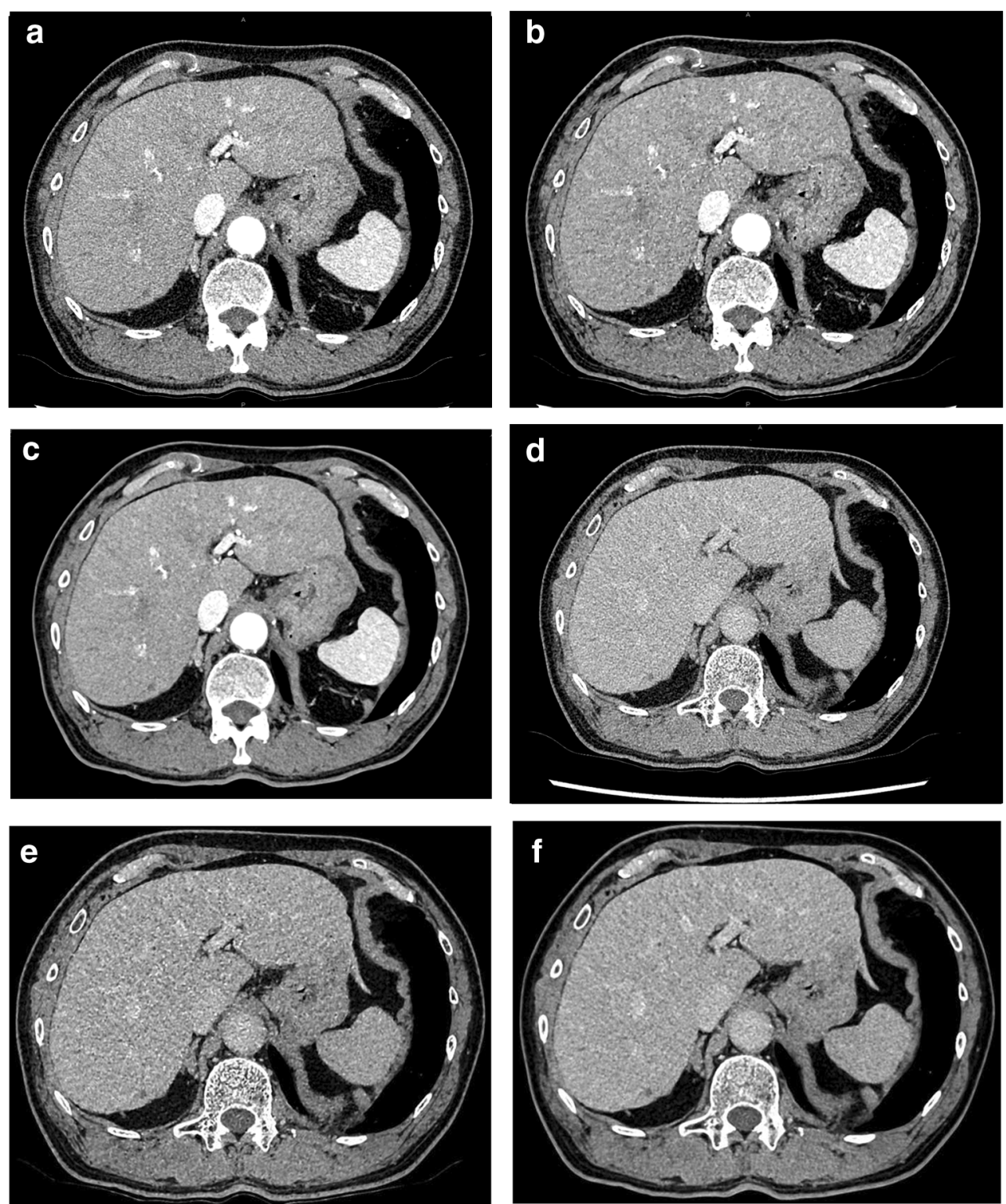

Fig. 3 Hepatic arterial (a-c) and equilibrium phase images (d-f) of a 76-year-old man. Reconstruction was with hybrid-IR (a, d), MBIR (b, e), and DLR $(\mathbf{c}, \mathbf{f})$. The image noise was lower on the DLR image than on the other images

Publisher's note Springer Nature remains neutral with regard to jurisdictional claims in published maps and institutional affiliations. 